\title{
Descriptive epidemiology of African horse sickness in Zimbabwe
}

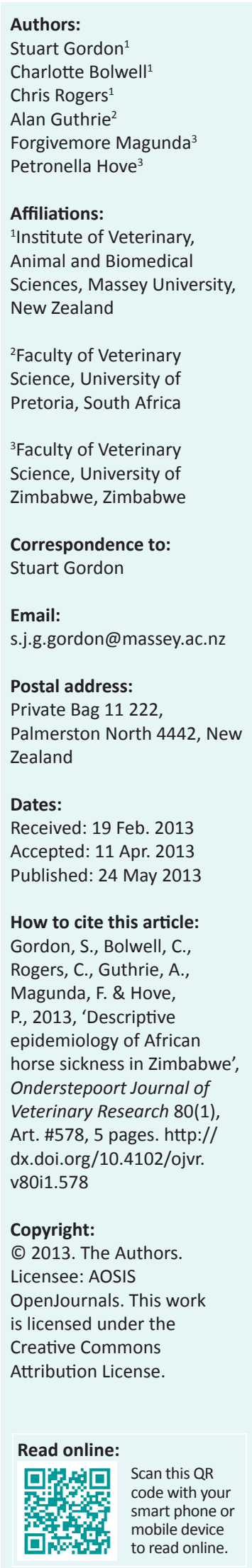

A study of the prevalence of African horse sickness in horses was conducted, using records from two private equine practices in Harare for the period 1998-2004. Results indicated a higher prevalence of the disease in horses in Zimbabwe in the late rainy season (March - May). Age of the horse was found to be a significant risk factor, with foals or yearlings appearing to be 1.80 times more likely to contract the disease compared with horses older than two years. The case fatality rate in foals or yearlings was also higher than in older age groups, but this difference was not significant. The vaccination status was an important risk factor, with vaccinated horses 0.12 times less likely to die from the disease compared with unvaccinated horses. Young, unvaccinated horses therefore seem to be the most susceptible to the disease and have greater chances of fatality. This study highlights the importance of adequately protecting horses against African horse sickness by providing immunisation through vaccination and discusses the need to review current vaccination strategies being practiced in Zimbabwe.

\section{Introduction}

African horse sickness (AHS) is an infectious and non-contagious disease, affecting all species of Equidae, caused by a double-stranded RNA orbivirus (Calisher \& Mertens 1998). AHS was first recognised in southern Africa, with the first major outbreak recorded in 1719, when more than 1700 animals died (Theiler 1921 in Mellor \& Hamblin 2004). Although endemic in Equidae in sub-Saharan Africa, outbreaks have also been recorded in north Africa, the middle and near east and southern Europe (MacLachlan \& Guthrie 2010). Both AHS and bluetongue viruses are transmitted by the biting midges Culicoides. Culicoides imicola is the field vector of AHS virus although Culicoides bolitinos has been shown to play an important role in the transmission of AHS virus in the cooler upper highlands of South Africa (Meiswinkel \& Paweska 2003). The highest incidence of the disease usually occurs in the late summer and early autumn in years when the climatic conditions favour an abundance of Culicoides midges (Coetzer \& Guthrie 2004).

The distribution of AHS virus is governed by a number of factors, including the efficiency of control measures, the presence of long-term vertebrate reservoirs and the occurrence of the Culicoides vectors (Mellor \& Hamblin 2004). It was shown by Wellby et al. (1996) and Mellor and Hamblin (2004) that the virogenesis and infection rates of the Culicoides vectors were directly proportional to an increase in temperature, but that the survival rates of Culicoides were inversely related to temperature. This suggests that transmission should be possible only at certain times of the year, giving the disease the same seasonality as the vector species. Previous studies conducted in Zimbabwe have shown the disease is usually diagnosed in late summer and early autumn, with most cases occurring towards the end of the rainy season (March - May) (Blackburn \& Swanepoel 1988a). In contrast, Henning (1956) found the disease occurred slightly earlier in South Africa, starting in February and declining sharply with the first frosts in May.

The relationship between the fatality rate in horses due to AHS compared with the age of the affected horse and the immunity status induced by vaccination was studied in Zimbabwe by Blackburn and Swanepoel (1988a). The authors reported 207 cases of clinically diagnosed AHS, with 107 deaths, between 1975 and 1980. Furthermore, 56 of these cases, with 50 deaths, were recorded in foals. Erasmus (1978) claimed, however, that the age, sex and body condition of the host animal have no influence on the susceptibility to AHS in naïve horses.

AHS cases have been recorded in horses that were vaccinated, suggesting that vaccination does not provide adequate protection (Blackburn \& Swanepoel 1988a). However, these findings were reported when the vaccine used in Zimbabwe contained only AHS virus serotypes one to six, unlike the freeze-dried, polyvalent, modified live, attenuated AHS virus vaccine (Onderstepoort Biological Products ${ }^{\circledR}$ ) used today. The current vaccine contains serotypes one to four and serotypes six to eight. Experimental studies have shown that antibody decline in foals with maternally derived immunity occurs between two and four months after birth (Blackburn \& 
Swanepoel 1988b). More recently, Crafford et al. (2013) reported that maternal antibodies to AHS virus acquired passively by foals born to vaccinated mares varied markedly, with further variation noted in the duration of passive immunity to individual AHS virus serotypes. This raises concern over the general belief amongst horse owners and equine veterinarians in Zimbabwe that foals with maternally derived immunity should only be vaccinated when between six and nine months of age.

Reliable data on AHS in horses are available readily from private practitioners, although, since 1998, reporting of the occurrence of this notifiable disease by veterinarians to government veterinary officers has been erratic (O. Kakono pers. comm., 09 November 2006). This has resulted in a gap in the knowledge about the disease status in the horse population across the country.

The objectives of this study were to determine the practice prevalence of AHS in the Harare region and the distribution of AHS cases by year, season, age, sex, and vaccination status. The effect of age and vaccination status on case fatality was also investigated.

\section{Materials and methods Selection of veterinary surgeries, data collection and analysis}

A convenience sample of two private veterinary surgeries in Harare was selected, based on reliable record keeping and a large equine caseload covering a wide practice area. Records of AHS cases available at the two private veterinary surgeries, for the period 1998-2004, were used for this study. The population of horses at risk was 16 987, as determined from the annual horse census of the Central Veterinary Laboratories of Zimbabwe and from data provided by the Horse Society of Zimbabwe, the Mashonaland Turf Club, the Thoroughbred Association of Zimbabwe, the Polo Association of Zimbabwe and the Polo $X$ Association of Zimbabwe. Horses included in this population were from the districts of Marondera, Harare and Goromonzi, which represented the practice area of the two veterinary practices.
Diagnosis of AHS was based on clinical signs and postmortem lesions recognised by the veterinarian or during laboratory diagnosis. The laboratory diagnosis was based on the antigen-capture enzyme-linked immunosorbent assay (ELISA) as described previously by Hamblin et al. (1991). No repeat cases of AHS in individual horses were recorded in any year.

A total of 8529 horses, representing $50.2 \%$ of the 16987 horses in the study area, were attended to by the two private practices during the study period. Of the 8529 horses attended to, 980 $(11.5 \%)$ were foals or yearlings ( $<$ two years of age).

Data were extracted from each case record, including the date of diagnosis, age, sex, vaccination status and disease outcome of each affected horse. The diagnosis date was used to determine the annual and monthly cases and the seasonality of the disease. The following seasonal categories were generated: early rainy season (December - February), late rainy season (March - May), cold dry season (June August), and the warm dry season (September - November). Age of the case horses was categorised into three groups: foals or yearlings (< two years of age), subadults (two to five years of age) and adults ( $>$ five years of age).

The annual practice prevalence and annual case fatality rates for AHS were calculated for the years 1998-2004. The practice prevalence and case fatality rates for AHS, stratified by season, year and age, were calculated over the whole study period. The effect of age on the odds of contracting AHS was evaluated by calculating the odds ratio (OR) and $95 \%$ confidence intervals (CI). The OR was also calculated for the effect of age and the effect of vaccination status on the odds of fatality due to AHS.

\section{Results}

The practice prevalence of AHS during the study period was $1.96 \%$ (167/8529). The average annual number of AHS cases recorded was 24 , with the highest practice prevalence recorded in 2003 and the lowest in 1998 (Table 1).

TABLE 1: The annual practice prevalence of African horse sickness in horses recorded by two Harare equine practices for the period $1998-2004$.

\begin{tabular}{|c|c|c|c|c|c|c|c|}
\hline Variable & Level & AHS cases & $\begin{array}{l}\text { Number of horses } \\
\text { seen by practices }\end{array}$ & $\begin{array}{l}\text { AHS prevalence } \\
(\%)\end{array}$ & $95 \% \mathrm{Cl} \dagger$ & AHS fatalities & $\begin{array}{l}\text { AHS case } \\
\text { fatality rate }\end{array}$ \\
\hline \multirow[t]{7}{*}{ Year } & 1998 & 9 & 1580 & 0.57 & $0.30-1.11$ & 6 & 66.7 \\
\hline & 1999 & 34 & 1381 & 2.46 & $1.77-3.42$ & 21 & 61.8 \\
\hline & 2000 & 47 & 1330 & 3.53 & $2.66-4.66$ & 15 & 31.9 \\
\hline & 2001 & 19 & 1230 & 1.54 & $0.99-2.39$ & 7 & 36.8 \\
\hline & 2002 & 10 & 1036 & 0.97 & $0.53-1.77$ & 3 & 30.0 \\
\hline & 2003 & 36 & 980 & 3.67 & $2.66-5.04$ & 12 & 33.3 \\
\hline & 2004 & 12 & 992 & 1.21 & $0.69-2.10$ & 3 & 25.0 \\
\hline \multirow[t]{4}{*}{ Season } & $\begin{array}{l}\text { Early rainy } \\
\text { (December - February) }\end{array}$ & 15 & 8529 & 0.18 & $0.11-0.30$ & - & - \\
\hline & $\begin{array}{l}\text { Late rainy } \\
\text { (March-May) }\end{array}$ & 115 & 8529 & 1.35 & $1.13-1.62$ & - & - \\
\hline & $\begin{array}{l}\text { Cold dry } \\
\text { (June - August) }\end{array}$ & 31 & 8529 & 0.36 & $0.25-0.51$ & - & - \\
\hline & $\begin{array}{l}\text { Warm dry } \\
\text { (September - November) }\end{array}$ & 6 & 8529 & 0.07 & $0.03-0.15$ & - & - \\
\hline Age & $>$ two years of age & 136 & 7549 & 1.80 & $1.52-2.13$ & 25 & 36.8 \\
\hline
\end{tabular}


Across all years, the highest numbers of AHS cases were recorded from March (16.2\%; 27/167) to June $(18.0 \%$; $30 / 167)$, with peak numbers reached in April $(21.0 \% ; 35 / 167)$ and May (31.7\%; 53/167). From July onwards, the number of cases decreased dramatically, with no cases recorded in August and November. The late rainy season recorded the highest number of cases $(68.9 \% ; 115 / 167)$, whilst the lowest number of cases $(3.6 \% ; 6 / 167)$ was recorded in the warm dry season (Table 1).

Male horses accounted for $48.5 \%(81 / 167)$ of the AHS cases recorded, whilst female horses accounted for $36.5 \%$ of the cases $(61 / 167)$. No sex identification was recorded in the medical records of 25 horses. The highest numbers of AHS cases were recorded in adult horses and subadults, with these groups accounting for $40.7 \%(68 / 167)$ of the total AHS cases recorded. The lowest number of cases $(18.6 \%$; 31/167) was recorded in foals or yearlings.

Foals or yearlings recorded a higher prevalence of AHS compared with horses older than two years (Table 1). The odds of AHS developing were 1.8 times greater in foals or yearlings (95\% CI: 1.3-2.9) compared with horses older than two years. The majority of the horses with AHS had previously been vaccinated against AHS (85\%; 142/167).

The average annual number of fatalities due to AHS was 9.6, with the highest case fatality rates recorded in 1998 and 1999 and the lowest in 2004 (Table 1). Foals or yearlings recorded the highest overall case fatality rate, followed by adults and subadults. The odds of foals or yearlings dying from AHS were 1.8 times greater (95\% CI: 0.90-4.30) compared with horses older than two years, although the odds were not significantly different. Unvaccinated horses recorded a higher overall case fatality rate compared with vaccinated horses (Table 1). Higher case fatality rates were recorded for unvaccinated foals or yearlings, subadults and adult horses compared with their respective vaccinated age categories (Table 2). Unvaccinated subadults had the highest case fatality rates, followed by unvaccinated adults and unvaccinated foals (Table 2). The odds of vaccinated horses dying from AHS were 0.12 times less likely (95\% CI: 0.044-0.350) compared with unvaccinated horses.

\section{Discussion}

The prevalence of AHS in Zimbabwe recorded during the present study was lower than in previous reports (Blackburn
1982; Blackburn \& Swanepoel 1988a; Musuka 1999). Blackburn and Swanepoel (1988a) recorded 207 cases of AHS from 1654 horses studied in Zimbabwe in 1980, representing a $12 \%$ prevalence compared to a $1.96 \%$ prevalence found in this study. This could possibly be attributed to improved vaccination and control measures practiced in the country during this current study period. It is also important to note that the prevalence of AHS recorded in this study may be an underestimation of true values, as results are based on a convenience sample taken from two veterinary surgeries only. Not all cases of AHS in the horse population, within the catchment area under study, may have been presented to these two surgeries.

The prevalence of AHS was higher in years that experienced early rains followed by a dry spell (rainfall data supplied by the Dept. of Meteorological Services, Harare) and there was a definite seasonal distribution, with most cases being diagnosed between late summer and autumn (February June). These findings concurred with observations from earlier studies in Zimbabwe (Blackburn \& Swanepoel 1988a).

The seasonality of the disease is also affected by vector patterns. The infection rates of the vector, Culicoides, and the rate of virogenesis increase with increasing environmental temperature although the rate of adult fly mortality may also increase (Mellor \& Hamblin 2004; Wellby et al. 1996). Culicoides imicola, however, breed only in wet or damp areas, which may explain why AHS cases are low in the warm, dry months despite high environmental temperatures favouring increased rates of virogenesis and infection (Baylis, Mellor \& Meiswinkel 1999).

It has been proposed that a strong link exsits between major outbreaks of AHS in southern Africa and the warm phase of the El Niño-Southern Oscillation (ENSO) (Baylis et al. 1999). It has been suggested that this link is due to the combination of heavy rain and drought that the ENSO brings to southern Africa, creating breeding conditions that allow the Culicoides vector to increase more than 200-fold (Baylis et al. 1999; Meiswinkel, Nevill \& Venter 1994; Mellor \& Hamblin 2004). The results of the present study support this suggestion, as the highest practice prevalence $(3.67 \%)$ was recorded in 2003 when a large ENSO was reported. The lowest practice prevalence $(0.57 \%)$ was recorded in 1998 when the ENSO was weak and a moderate La Niña-Southern Oscillation was reported (National Institute of Water and Atmospheric Research 2013). Furthermore, it has been predicted by

TABLE 2: Case fatality rates for African horse sickness in vaccinated and unvaccinated horses according to age group.

\begin{tabular}{|c|c|c|c|c|c|}
\hline Age group & Vaccination status & Total number of cases & Total fatalities & Case fatality rate (\%) & $95 \% \mathrm{Cl}+$ \\
\hline \multirow[t]{2}{*}{ Foals or yearlings } & Unvaccinated & 3 & 2 & 67 & $20.8-93.9$ \\
\hline & Vaccinated & 28 & 14 & 50 & $32.6-67.4$ \\
\hline \multirow[t]{2}{*}{ Subadults } & Unvaccinated & 8 & 7 & 88 & $52.9-97.8$ \\
\hline & Vaccinated & 60 & 18 & 30 & $19.9-42.5$ \\
\hline \multirow[t]{2}{*}{ Adults } & Unvaccinated & 14 & 11 & 79 & $52.4-92.4$ \\
\hline & Vaccinated & 54 & 15 & 28 & $17.6-40.9$ \\
\hline
\end{tabular}

$\mathrm{Cl}$, confidence interval.

Cl, confidence interval.
$\dagger$, Lowry, R., 2013, 'The confidence interval of a proportion', in VassarStats: Website for Statistical Computation, viewed 11 February 2013, from http://www.vassarstats.net/prop1.html 
Wittmann and Baylis (2000) that the distribution of C. imicola will become more widespread with increasing global temperatures, bringing the AHS virus into the range of other Culicoides species that are known to be competent vectors of this disease.

The results of the present study showed that the age of the horse was associated with the occurrence of AHS in this population, with foals or yearlings having greater odds of developing the disease than older horses. These findings concur with earlier observations in Zimbabwe (Blackburn \& Swanepoel 1988a), although Erasmus (1978) claimed that age had no influence on susceptibility to the disease. As supported by the findings of this study, age susceptibility and survival may be linked to the immune status of a horse due to vaccination. The response to vaccination is therefore important, as the immune status of the horse modifies the form of the disease and thus the associated mortality. However, despite high levels of horse vaccination in Zimbabwe, a high prevalence of AHS was recorded. A high prevalence of AHS in vaccinated horses in the country was also reported previously (Blackburn \& Swanepoel 1988a). The polyvalent vaccine in use at the time of that study (1980) included attenuated strains of serotypes one to six only (Blackburn \& Swanepoel 1988a). In the present study, however, cases still occurred in vaccinated horses, although the polyvalent vaccine used now also includes serotypes seven and eight. Furthermore, Weyer et al. (2013) showed that horses immunised against AHS virus can be infected both clinically and subclinically with AHS virus following natural infection in field conditions, with $16 \%$ of immunised horses being infected over a two-year period.

The higher case fatality rates recorded in foals or yearlings may be due to incomplete protection against the AHS virus after the initial vaccination, which has been attributed to interference between the virus serotypes (Coetzer \& Guthrie 2004). Some strains of vaccine may also be immunogenically weak and several courses of vaccination may be required to achieve full immunity to the AHS virus, thus making foals or yearlings that have not yet received multiple vaccinations more susceptible (Laegreid 1996). Experimental studies conducted by Blackburn and Swanepoel (1988a) showed that multiple vaccinations with attenuated strains of AHS serotypes one to six led to a broader response to various serotypes and to a higher individual serotype titre. However, it has been highlighted that although protective responses to all the virus serotypes in the polyvalent vaccine may be achieved only after repeated immunisation, excessive administration of vaccine over many years appears to lead to a state of immunological unresponsiveness or hypersensitivity (Erasmus 1978). This could explain why there is often an increase in cases of AHS in older horses. Improper storage of the vaccine or incorrect administration of the vaccine may also contribute to vaccine failure.

Crafford et al. (2013) reported a mean half-life of only 20.5 days for passively acquired maternal neutralising antibody to AHS virus recorded in six foals in an endemic area in the Western Cape province of South Africa. Blackburn and Swanepoel (1988b) showed that passively acquired antibodies in foals against individual AHS serotypes declined to undetectable levels between two and four months of age. Furthermore, the vaccination of foals between three and four months of age resulted in a weak antibody response, which did not adversely affect pre-existing low levels of maternal antibody (Blackburn \& Swanepoel 1988b). Thus, foals from vaccinated dams may succumb to AHS as early as at three months of age and immunisation at an earlier age could therefore help to control foal susceptibility to and mortality from AHS. The effectiveness of the current vaccination strategies against AHS used in Zimbabwe may, therefore, require revision. The vaccination of younger foals with maternally derived antibody against some serotypes of AHS could be considered instead of the current practice of vaccinating foals only older than six months in Zimbabwe.

The sex of the horse was not adequately investigated as a possible risk factor as the sex composition of the original population was unknown, thus necessitating the need for further studies in this area.

\section{Conclusion}

The age of the horse was found to be significantly associated with AHS, with foals or yearlings more likely to contract the disease compared with horses older than two years. The AHS case fatality rate in foals or yearlings was also found to be higher than in older age groups. Furthermore, the vaccination status was found to be an important risk factor, with vaccinated horses less likely to die from AHS compared with unvaccinated horses.

It appears, therefore, that young, unvaccinated horses seem to be the most susceptible to AHS and that they suffer greater chances of fatality. This reinforces the importance of adequately protecting horses against AHS through vaccination, highlighting the need to review current vaccination strategies being practised in Zimbabwe.

\section{Acknowledgements}

The authors would like to acknowledge the cooperation and support of the two private practices in Harare used for data collection in this study. The authors are also grateful for the information provided by the Central Veterinary Laboratories of Zimbabwe, the Horse Society of Zimbabwe, the Mashonaland Turf Club, the Thoroughbred Association of Zimbabwe, the Polo Association of Zimbabwe and the Polo X Association of Zimbabwe.

\section{Competing interests}

The authors declare that they have no financial or personal relationship(s) that may have inappropriately influenced them in writing this article. 


\section{Authors' contributions}

S.G. (Massey University) wrote the manuscript and acted as project leader. P.H. (University of Zimbabwe) and F.M. (University of Zimbabwe) designed the project and collected the data from the medical records at the two private equine practices in Harare. C.B. (Massey University), C.R. (Massey University) and A.G. (University of Pretoria) made conceptual contributions and assisted with the data analysis, statistical work and editing of the final manuscript.

\section{References}

Baylis, M., Mellor, P.S. \& Meiswinkel, R., 1999, 'Horse sickness and ENSO in South Africa', Nature 397, 597.

Blackburn, N.K., 1982, 'Studies on African horse sickness in Zimbabwe', D.Phil thesis, Faculty of Medicine, University of Zimbabwe.

Blackburn, N.K. \& Swanepoel, R., 1988a, 'African horse sickness in Zimbabwe: 1972 to 1981', Tropical Animal Health Production 20, 169-176.

Blackburn, N.K. \& Swanepoel, R., 1988b, 'Observation on antibody levels associated with active and passive immunity to African horse sickness', Tropical Animal Health Production 20, 203-210.

Calisher, C.H. \& Mertens, P.P.C., 1998, 'Taxonomy of horse sickness viruses', Archives of Virology Supplement 14, 3-11.

Coetzer J.A.W. \& Guthrie A.J., 2004, 'African horse sickness', in J.A.W. Coetzer \& R.C. Tustin (eds.), Infectious diseases of livestock, 2 nd edn., Oxford University Press Southern Africa, Cape Town, pp. 1231-1246.

Crafford, J.E., Lourens, C.W., Gardner, I.A., Maclachlan, N.J. \& Guthrie, A.J., 2013, 'Passive transfer and rate of decay of maternal antibody against African horse sickness virus in South African Thoroughbred foals', Equine Veterinary Journal 45 n.p. http://dx.doi.org/10.1111/evj.12015

Erasmus, B.J., 1978, 'A new approach to polyvalent immunization against African horsesickness', in J.T. Bryans \& H. Gerber (eds.), Proceedings of the 4th International Conference on Equine Infectious Diseases, Veterinary Publications, Princeton, pp. 401-403.
Hamblin, C., Mertens, P.P.C., Mellor, P.S., Burroughs, J.N. \& Crowther, J.R., 1991, 'A serogroup specific enzyme-linked immunosorbent assay for the detection of African horse sickness viruses', Journal of Virological Methods 31, 285-292.

Henning, M.W., 1956, Animal diseases of southern Africa, 3rd edn., Central News Agency, Pretoria.

Laegreid, W.W., 1996, 'African horse sickness', in M.J. Studdert (ed.), Virus infections of equines, Elsevier, Amsterdam, pp. 101-123.

Lowry, R., 2013, 'The confidence interval of a proportion', in VassarStats: Website for Statistical Computation, viewed 11 February 2013, from http://www.vassarstats. net/prop1.html

MacLachlan, N.J. \& Guthrie, A.J., 2010, 'Re-emergence of bluetongue, African horse sickness, and other Orbivirus diseases', Veterinary Research 41, 35.

Meiswinkel, R., Nevill, E.M. \& Venter, G.J., 1994, 'Vectors: Culicoides spp.', in J.A.W. Coetzer, G.R. Thomson \& R.C. Tustin (eds.), Infectious diseases of livestock with special reference to southern Africa, Oxford University Press, Cape Town, pp. special

Meiswinkel, R. \& Paweska, J.T., 2003, 'Evidence for a new field Culicoides vector of African horse sickness in South Africa', Preventative Veterinary Medicine 60 African h3-253.

Mellor, P.S. \& Hamblin, C., 2004, 'African horse sickness', Veterinary Research 35, $445-466$.

Musuka, G.N., 1999, 'Culicoides biting midges, vectors of arboviruses in Zimbabwe', M.Phil thesis, University of Hertfordshire.

National Institute of Water and Atmospheric Research, 2008, Education and Training: El Niño and La Niña, viewed 07 February 2013, from http://www.niwa.co.nz/ education-and-training/schools/students/enln

Wellby, M.P., Baylis, M., Rawlings, P. \& Mellor, P.S., 1996, 'Effect of temperature on survival and rate of virogenesis of African horse sickness virus in Culicoides variipennis sonorensis (Diptera: Ceratopogonidae) and its significance in relation to the epidemiology of the disease', Bulletin of Entomological Research 86, 715720.

Weyer, C.T., Quan, M., Joone, C., Lourens, C.W., Maclachlan, N.J. \& Guthrie, A.J., 2013, 'African horse sickness in naturally infected, immunised horses', Equine Veterinary Journal 45, 117-119.

Wittmann, E. \& Baylis, M., 2000, 'Climate change: Effects on Culicoides-transmitted viruses and implications for the UK', Veterinary Journal 160, 107-117. 